\title{
Estratégia Heurística de Baixa Complexidade Aplicada à Decodificação Multiusuário em Sistemas DS/CDMA
}

\author{
Fernando Ciriaco, Wagner Okano, Taufik Abrão, Paul Jean E. Jeszensky
}

\begin{abstract}
Resumo-Este trabalho analisa o desempenho do algoritmo heurístico de busca local (LS - Local Search) baseado na distância Euclidiana mínima (1-opt LS) aliado ao detector de Viterbi, quando aplicados ao problema da detecção multiusuário (MuD - Multiuser Detection) em sistemas DS/CDMA (Direct Sequence/Code Division Multiple Access) codificados, sujeitos a canais com desvanecimento Rayleigh plano. A eficiência da estratégia proposta é analisada considerando-se o compromisso desempenho versus acréscimo na complexidade computacional e requisito de memória, quando comparado à estratégia de detecção baseada somente no detector de Viterbi. Por fim, apresenta-se este acréscimo nos requisitos de memória e de processamento para se alcançar o desempenho, em termos de taxa de erro de bit (BER - Bit Error Rate), do decodificador de máxima verossimilhança (ML - Maximum Likelihood).
\end{abstract}

Palavras-Chave-Algoritmos de Busca Local, Código Convolucional, DS/CDMA, Deteç̧ão Multiusuário, Canais Rayleigh Plano.

Abstract-The characteristics of the heuristic algorithms based on local search (LS), applied to a DS/CDMA (Direct Sequence/Code Division Multiple Access) coded multi-user detection (MuD) problem in Flat Rayleigh channels are introduced. The algorithms performance versus the increment in the computational complexity and memory requirement are carried out and the efficiency of the algorithm is compared through this performance tradeoff, shown these necessary system requirements to achieve the Bit Error Rate performance of the maximum likelihood detector (ML).

Keywords-Local search algorithms, Convolutional Code, DS/CDMA, Multiuser Detection, Flat Rayleigh Channels.

\section{INTRODUÇÃO}

O desvanecimento e a interferência de múltiplo acesso (MAI - Multiple Access Interference) são os dois principais fatores que limitam o desempenho de sistemas DS/CDMA. Desta forma, detectores multiusuário são utilizados para combater a MAI, enquanto a diversidade de percurso e a espaço-temporal usualmente são utilizadas no combate ao desvanecimento em

Fernando Ciriaco é aluno do programa de Doutorado da Escola Politécnica da Universidade de São Paulo e Coordenador do Curso de Engenharia Elétrica da Faculdade Pitágoras - Campus Londrina; fciriaco@lcs.poli.usp.br

Wagner Okano é Engenheiro de Telecomunicações da Sercomtel Celular S.A. Atualmente é estudante do programa de mestrado do Departamento de Engenharia Elétrica da Universidade Estadual de Londrina; wjokano@sercomtel.com.br.

Taufik Abrão é Professor Associado do Departamento de Engenharia Elétrica da Universidade Estadual de Londrina; taufik@uel.br

Paul Jean E. Jeszensky é Professor Titular do Departamento de Engenharia de Telecomunicações e Controle da Escola Politécnica da Universidade de São Paulo; pjj@lcs.poli.usp.br canais de comunicação sem fio. Adicionalmente, códigos corretores de erro no receptor (FEC - Forward Error Correction) são utilizados no combate da MAI e do desvanecimento de forma conjunta.

Portanto, a codificação é freqüentemente usada em sistemas de comunicação digital para proteger a informação do ruído e da interferência propiciando, desta maneira, uma menor degradação de desempenho no receptor. Para isso, utiliza-se a inserção de bits de redundância no sinal de informação, implicando em uma redução na taxa líquida de transmissão ou um aumento na largura de banda ocupada.

O sinal recebido por um detector convencional (CD Conventional Detector) DS/CDMA, constituído de um banco de filtros casados (MFB - Matched Filter Bank) às respectivas seqüências de espalhamento, não pode ser recuperado de forma ótima, pois é afetado pela MAI, pelo desvanecimento e pelo efeito near-far, resultando em um sistema cuja capacidade está bem abaixo da capacidade do canal [1]. O detector DS/CDMA convencional (MFB) também é conhecido como estratégia single-user de detecção.

Desta forma, uma grande variedade de detectores multiusuário foram propostos na literatura nas últimas duas décadas com a finalidade de reduzir a degradação de desempenho imposta pelo detector convencional. Em [1] demonstra-se que o melhor desempenho possível é alcançado com o uso de um detector baseado no critério de máxima verossimilhança (ML). Este detector procura estimar o vetor transmitido de forma a maximizar a probabilidade deste ter sido enviado, sendo que o procesamento do sinal estende-se por toda a mensagem, considerando a hipótese de que todas as mensagens transmitidas são equiprováveis. Porém, o detector ML possui uma complexidade computacional que cresce exponencialmente com o número de usuários, tornando inviável sua implementação [1]. Portanto, faz-se necessário a realização de investigações na área de detecção multiusuário sub-ótima, objetivando atender aos critérios de alto desempenho e baixa complexidade.

$\mathrm{Na}$ literatura, apesar de existirem vários trabalhos empregando procedimentos aproximativos para a detecção multiusuário, a maioria das investigações não considera sistemas codificados e/ou estão restritos a canais síncronos com ruído puramente aditivos (AWGN - Additive White Gaussian Noise) [2]-[6]. Diferentemente, este trabalho considera um sistema DS/CDMA codificado, sujeito a canais com desvanecimento, sendo analisado e proposto uma estratégia de detecção multiusuário sub-ótima utilizando o algoritmo de busca local 1-opt 
(1-optimum Local Search).

A seguir, a seção II descreve o modelo matemático do sistema DS/CDMA codificado em canal Rayleigh Plano síncrono. $\mathrm{Na}$ seção III é descrito o problema combinatório para a detecção multiusuário ótima, evidenciando a aplicabilidade de métodos combinatórios sub-ótimos. A característica e a descrição do algoritmo heurístico de busca local utilizado são apresentadas na seção IV. Resultados numéricos de simulação Monte-Carlo são discutidos na seção V. Na seção VI apresenta-se o incremento no número de operações e no requisito de memória pela utilização da técnica proposta quando comparado a decodificação de Viterbi. Por fim, na seção VII são apresentadas as principais conclusões desta investigação.

\section{Modelo do Sistema}

O transmissor, canal e receptor para o sistema DS/CDMA síncrono codificado são esquematizados na Fig. 1. O receptor é constituído, além do decodificador de Viterbi, de um algoritmo heurístico baseado na distância Euclidiana mínima, com o intuito de maximizar o desempenho do sistema em termos da taxa de erro de bits $(B E R)$, admitindo-se, somente, um acréscimo marginal em sua complexidade.

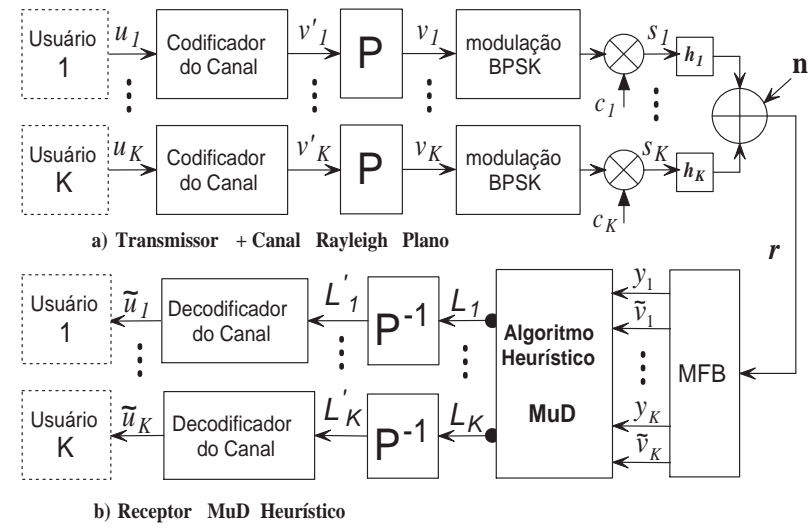

Fig. 1. (a) Modelo de transmissão e (b) recepção do sistema.

Este modelo considera transmissões do enlace reverso (UL uplink), onde cada um dos $K$ usuários (MS - Mobile Stations) transmitem para a estação base (BS - Base Station) utilizando uma simples antena na transmissão e na recepção.

A informação binária do $k$-ésimo usuário, de comprimento $N_{u},\left\{u_{k}[i] ; i=1, \ldots, N_{u}\right\}$, passa inicialmente por um codificador de canal, sendo adotado neste trabalho a codificação convolucional (CC - Convolutional Code) de taxa $R_{c}=$ $N_{u} / N_{c}$, fornecendo, na saída do codificador, uma seqüência de bits codificada $\left\{v_{k}^{\prime}[i] ; i=1, \ldots, N_{c}\right\}$, de comprimento $N_{c}$ bits. Esta seqüência passa então por um entrelaçador aleatório, denotado pelo operador $\mathrm{P}$, gerando uma sequiência de bits entrelaçada $\left\{v_{k}[i] ; i=1, \ldots, N_{c}\right\}$. Em seguida, as informações codificadas dos $K$ usuários são repassadas individualmente aos respectivos moduladores por deslocamento de fase binária (BPSK - Binary Phase Shift Keying). Essas informações codificadas são então espalhadas espectralmente, utilizando a técnica de sequência direta (DS/CDMA), através de uma sequência específica por usuário $c_{k}$, de comprimento $N_{s}$ chips. Uma portadora de frequência $f_{c}$ é utilizada para obter o sinal banda-passante. Assim, o sinal transmitido do $k$-ésimo usuário pode ser expresso como:

$$
\mathbf{s}_{k}(t)=\sqrt{2} v_{k} c_{k}(t) \cos \left(2 \pi f_{c} t+\phi_{k}\right)
$$

onde considerou-se cada usuário com potência de transmissão unitária e $\phi_{k}$ como sendo o ângulo de fase da portadora; omitiu-se o índice de bit $i$ por razões de concisão.

O sinal modulado $\mathbf{s}_{k}$ propaga-se, então, a partir da antena de transmissão do $k$-ésimo usuário no canal uplink até a antena receptora da estação-base sujeito a um canal com desvanecimento Rayleigh plano, de resposta $h_{k}$, o qual é assumido constante durante a duração do símbolo codificado. Desta forma, durante o $n_{s}$-ésimo intervalo de chip, de duração $T_{s}$, com $n_{s}=1, \ldots, N_{s}$, o sinal recebido $r_{n_{s}}$ pode ser considerado uma superposição dos sinais transmitidos a partir de $K \mathrm{MSs}$, sendo corrompido também pelo AWGN. Com isso, durante um intervalo de símbolo qualquer, o vetor de sinal recebido em banda-base, de dimensão $N_{s} \times 1$, pode ser expresso, em notação vetorial, como:

$$
\mathbf{r}=\mathbf{C H v}+\mathbf{n}
$$

onde $\mathbf{C}$ corresponde a matriz formada pelas sequências de espalhamento, de dimensão $N_{s} \times K ; \mathbf{H}$ corresponde a matriz de estimativas complexas da função de transferência do canal (CHTF - CHannel Transfer Function), de dimensão $K \times K$; v corresponde ao vetor sinal transmitido equivalente em bandabase relativo aos $K$ usuários, de dimensão $K \times 1$ e o termo n corresponde ao vetor de ruído AWGN amostrado, de média zero, variância $\sigma_{n}^{2}=N_{0} / 2$ e dimensão $N_{s} \times 1$, sendo definidos como:

$$
\begin{aligned}
\mathbf{r} & =\left[\begin{array}{lll}
r_{1} & r_{2} \ldots & r_{N_{s}}
\end{array}\right]^{T}, \\
\mathbf{H} & =\operatorname{diag}\left[\begin{array}{lll}
h_{1} & h_{2} \ldots & h_{K}
\end{array}\right], \\
\mathbf{v} & =\left[\begin{array}{lll}
v_{1} & v_{2} \ldots & v_{K}
\end{array}\right]^{T}, \\
\mathbf{n} & =\left[\begin{array}{llll}
n_{1} & n_{2} \ldots & n_{N_{s}}
\end{array}\right]^{T}, \\
\mathbf{C} & =\left[\begin{array}{cccc}
c_{11} & c_{21} & \ldots & c_{K 1} \\
c_{12} & c_{22} & \ldots & c_{K 2} \\
\vdots & \vdots & \ddots & \vdots \\
c_{1 N_{s}} & c_{2 N_{s}} & \cdots & c_{K N_{s}}
\end{array}\right] .
\end{aligned}
$$

Por simplificação, assume-se que as CHTFs são perfeitamente estimadas na BS. Com isso, a saída do filtro casado (MF - matched filter) correspondente aos $K$ usuários, considerando o uso do combinador de razão máxima (MRC - maximal ratio combiner) pode ser expressa como [7], [8]:

$$
\begin{aligned}
\mathbf{y} & =\mathbf{C H}{ }^{H} \mathbf{R}=\mathbf{H}^{H} \mathbf{C}^{T} \mathbf{C H} \mathbf{v}+\mathbf{H}^{H} \mathbf{C}^{T} \mathbf{n} \\
& =\mathbf{R} \mathbf{v}+\tilde{\mathbf{n}}
\end{aligned}
$$

onde: $\mathbf{y}=\left[\begin{array}{llll}y_{1} & y_{2} \ldots & y_{K}\end{array}\right]^{T}$,

$\mathbf{R}=\mathbf{H}^{H} \mathbf{C}^{T} \mathbf{C H}$

$\tilde{\mathbf{n}}=\mathbf{H}^{H} \mathbf{C}^{T} \mathbf{n}$.

Considerando decisão abrupta sobre a saída do MFB, obtém-se uma estimativa do sinal transmitido:

$$
\tilde{\mathbf{v}}=\operatorname{sign}[\mathbf{y}]
$$


onde $\operatorname{sign}(x)=x / \operatorname{abs}(x)$, admitindo $x \in \mathbb{R}$.

Desta forma, maximizar o desempenho consiste na maximização da probabilidade a posteriori $p(\tilde{\mathbf{v}} \mid \mathbf{r}, \mathbf{H})$, baseado na observação do vetor sinal recebido, r, da matriz CHTF, $\mathbf{H}$, e do sinal à saída do MFB, $\tilde{\mathbf{v}}$. Usando a regra de Bayes, a probabilidade a posteriori é expressa por [9]:

$$
P\left(\tilde{\mathbf{v}}_{i} \mid \mathbf{r}, \mathbf{H}\right)=\frac{p\left(\mathbf{r} \mid \tilde{\mathbf{v}}_{i}, \mathbf{H}\right) P\left(\tilde{\mathbf{v}}_{i}\right)}{p(\mathbf{r})}, \quad i=1,2, \ldots 2^{K}
$$

onde $p\left(\mathbf{r} \mid \tilde{\mathbf{v}}_{i}, \mathbf{H}\right)$ é a função densidade de probabilidade condicional (PDF - probability density function) do vetor sinal recebido $\mathbf{r}$, dado que $\tilde{\mathbf{v}}_{i}$ foi transmitido e $P\left(\tilde{\mathbf{v}}_{i}\right)$ é a probabilidade a priori da $i$-ésima combinação do vetor de bits transmitido considerado. Assim, a estimativa suave para o bit decodificado pode ser expressa em termos da razão de verossimilhança logarítmica (LLR - Log-Likelihood Ratio) associada ao $k$-ésimo usuário, como [10]:

$$
L_{k}=\log \frac{P\left(v_{k}=+1 \mid \mathbf{r}, \mathbf{H}\right)}{P\left(v_{k}=-1 \mid \mathbf{r}, \mathbf{H}\right)}
$$

Note-se que a probabilidade $P\left(v_{k}=b \in\{ \pm 1\} \mid \mathbf{r}, \mathbf{H}\right)$ é obtida através de uma soma de probabilidades $P(\tilde{\mathbf{v}} \mid \mathbf{r}, \mathbf{H})$ para todos os $\tilde{\mathbf{v}} \in \mathcal{V}_{b}$, considerando o $k$-ésimo bit $b$. Deste modo, a eq. (14) pode ainda ser expressa como:

$$
L_{k}=\log \frac{\sum_{\tilde{\mathbf{v}} \in \mathcal{V}_{+1}} P(\tilde{\mathbf{v}} \mid \mathbf{r}, \mathbf{H})}{\sum_{\tilde{\mathbf{v}} \in \mathcal{V}_{-1}} P(\tilde{\mathbf{v}} \mid \mathbf{r}, \mathbf{H})}
$$

Sabe-se também que o denominador da eq. (13) pode ser expresso por [9]:

$$
p(\mathbf{r})=\sum_{i=1}^{2^{K}} p\left(\mathbf{r} \mid \tilde{\mathbf{v}}_{i}, \mathbf{H}\right) P\left(\tilde{\mathbf{v}}_{i}\right)
$$

sendo independente de uma combinação particular para o $i$-ésimo vetor de bits (dimensão $K \times 1$ ) transmitido ou para a $i$-ésima sequência específica considerando $i=1, \ldots, 2^{K}$.

Portanto, se as probabilidades a priori $P\left(\tilde{\mathbf{v}}_{i}\right)$ são iguais para todas as sequências, então a eq. (14) pode ser expressa por:

$$
L_{k}=\log \frac{\sum_{\tilde{\mathbf{v}} \in \mathcal{V}_{+1}} p(\mathbf{r} \mid \tilde{\mathbf{v}}, \mathbf{H})}{\sum_{\tilde{\mathbf{v}} \in \mathcal{V}_{-1}} p(\mathbf{r} \mid \tilde{\mathbf{v}}, \mathbf{H})}
$$

Observe-se que na eq. (2), r é uma amostra aleatória da variável complexa multi-dimensional com distribuição Gaussiana, no qual o valor médio do vetor é $\mathbf{C H v}$ e a matriz covariância, dimensão $N_{s} \times N_{s}, \mathbf{R}_{\mathbf{n}}$ é dada por [7]:

$$
\mathbf{R}_{\mathbf{n}}=\mathbb{E}\left\{\mathbf{n n}^{H}\right\}=\sigma_{n}^{2} \mathbf{I}
$$

sendo I a matriz identidade.

Assumindo que as amostras de ruídos na BS (a cada $T_{s}$ ) sejam descorrelacionados, a referida distribuição Gaussiana complexa multi-variável pode ser descrita por [11]:

$$
p(\mathbf{r} \mid \tilde{\mathbf{v}}, \mathbf{H})=\frac{\exp \left\{-(\mathbf{r}-\mathbf{C H} \tilde{\mathbf{v}})^{H} \mathbf{R}_{\mathbf{n}}{ }^{-1}(\mathbf{r}-\mathbf{C H} \tilde{\mathbf{v}})\right\}}{{\sqrt{2 \pi\left|\mathbf{R}_{\mathbf{n}}\right|^{N}}}^{N_{s}}}
$$

Por fim, da eq. (18) e eq. (19), obtêm-se [9]:

$$
p(\mathbf{r} \mid \tilde{\mathbf{v}}, \mathbf{H})=\frac{1}{{\sqrt{2 \pi \sigma_{n}^{2}}}^{N_{s}}} \exp \left\{-\frac{1}{2 \sigma_{n}^{2}}\|(\mathbf{r}-\mathbf{C H} \tilde{\mathbf{v}})\|^{2}\right\}
$$

Deste modo, a eq. (17) pode ainda ser expressa como:

$$
L_{k}=\log \frac{\sum_{\tilde{\mathbf{v}} \in \mathcal{V}_{+1}} \frac{1}{{\sqrt{2 \pi \sigma_{n}^{2}}}^{N_{s}}} \exp \left\{-\frac{1}{2 \sigma_{n}^{2}}\|(\mathbf{r}-\mathbf{C H} \tilde{\mathbf{v}})\|^{2}\right\}}{\sum_{\tilde{\mathbf{v}} \in \mathcal{V}_{-1}} \frac{1}{{\sqrt{2 \pi \sigma_{n}^{2}}}^{N_{s}}} \exp \left\{-\frac{1}{2 \sigma_{n}^{2}}\|(\mathbf{r}-\mathbf{C H} \tilde{\mathbf{v}})\|^{2}\right\}}
$$

Note-se que o universo de busca do numerador e denominador da eq. (21) possui dimensão $2^{K-1}$, ou seja, cresce exponencialmente com o número de usuários.

\section{DESCRIÇÃo DO PROBLEMA}

Em 1998, Verdú [1] demostrou que a solução ótima para recuperar os bits de informação consiste no uso do detector de máxima verossimilhança. Com isso, o detector multiusuário ótimo (OMuD - optimum multiuser detector) consiste na busca do melhor vetor de bits de dados em um conjunto com todas as possibilidades, ou seja, $2^{K-1}$ vetores de bits candidatos, o qual apresenta crescimento exponencial com o número de usuários, tornando-o impraticável em sistemas com elevado número de usuários. Na literatura, este problema é conhecido como de combinação NP-completo $^{1}$, no qual os algoritmos tradicionais de otimização combinatória são ineficientes. Em função da alta complexidade do detector $\mathrm{OMuD}$, vários trabalhos foram propostos visando a manutenção de desempenho próximo ao ótimo, aliado a uma redução substancial de complexidade. Neste contexto, os métodos heurísticos mostram-se como soluções atraentes, pois são ferramentas de otimização baseados na aproximação progressiva de um dado problema.

Genericamente, sob a restrição de um espaço de busca e, portanto, sob a condição de redução de complexidade, todos os algoritmos heurísticos procuram por melhores soluções seguindo uma função custo (fitness value), a qual quantifica a aptidão das possíveis soluções. Através do conhecimento dessa medida de aptidão, a procura é então direcionada para as regiões do universo de busca onde a probabilidade de se encontrar a solução ótima para o problema seja maior. A reduzida complexidade resultante, aliada à boa qualidade das soluções encontradas, proporcionam às técnicas heurísticas aplicações em diversos problemas que envolvem otimização combinatória [13].

A função custo para o contexto da detecção multiusuário é baseada na função de verossimilhança (LLF - Log-Likelihood Function), eq. (20), considerando a distância Euclidiana do termo $\|(\mathbf{r}-\mathbf{C H} \tilde{\mathbf{v}})\|^{2}$, podendo ser escrita como [7], [9]:

$$
D(\tilde{\mathbf{v}})=2 \Re\left(\tilde{\mathbf{v}}^{H} \mathbf{y}\right)-\tilde{\mathbf{v}}^{H} \mathbf{R} \tilde{\mathbf{v}}
$$

No intuito de evitar o aumento exponencial da complexidade computacional, os somatórios no numerador e no denominador da eq. (21) são substituídos pelo seu termo dominante, tal como sugerido em [7], [8], resultando na aproximação:

$$
\begin{aligned}
L_{k} & \approx-\frac{1}{2 \sigma_{n}^{2}}\left(\left\|\left(\mathbf{r}-\mathbf{C H} \tilde{\mathbf{v}}^{+}\right)\right\|^{2}-\left\|\left(\mathbf{r}-\mathbf{C H} \tilde{\mathbf{v}}^{-}\right)\right\|^{2}\right) \\
& \approx-\frac{1}{2 \sigma_{n}^{2}}\left(D\left(\tilde{\mathbf{v}}^{+}\right)^{2}-D\left(\tilde{\mathbf{v}}^{-}\right)^{2}\right)
\end{aligned}
$$

${ }^{1} \mathrm{NP}$ - nondeterministic polynomial time [12], não podendo ser resolvido em tempo computacional factível por ser de ordem não polinomial. Neste caso específico, a ordem cresce exponencialmente com o aumento do número de usuários. 
Desta forma, cada algoritmo heurístico aplicado ao problema da detecção multiusuário em sistemas DS/CDMA codificados busca maximizar a eq. (22) através de um vetor de bits candidato, denominado $\tilde{\mathbf{v}}$, cujo desempenho médio correspondente aproxima-se daquele obtido por um detector ML.

\section{Algoritmos Heurísticos}

Em comparação com as técnicas exatas os algoritmos heurísticos não garantem encontrar uma solução ótima após atingirem um critério de parada, mas estes têm demonstrado alta eficiência em problemas de larga combinação para casos práticos, além de poderem ser modificados facilmente, adaptando-se ao problema analisado [4], [6], [13]. Este trabalho utiliza o algoritmo heurístico de busca local 1-opt LS, pois este apresenta uma estratégia singela de procura e, conseqüentemente, uma reduzida complexidade computacional quando comparado aos demais métodos heurísticos.

\section{A. Algoritmo de Busca Local 1-opt}

O 1-opt LS pertence a uma classe de algoritmos de busca determinística, pois procura por uma solução ao redor de uma vizinhança baseada na distância de Hamming [4], [14]. Especificamente, para o algoritmo 1-opt LS, a vizinhança é composta por todos os possíveis candidatos com distância de Hamming igual a 1 da solução atual. O pseudo-código para o algoritmo 1-opt LS é apresentado no Algoritmo 1.

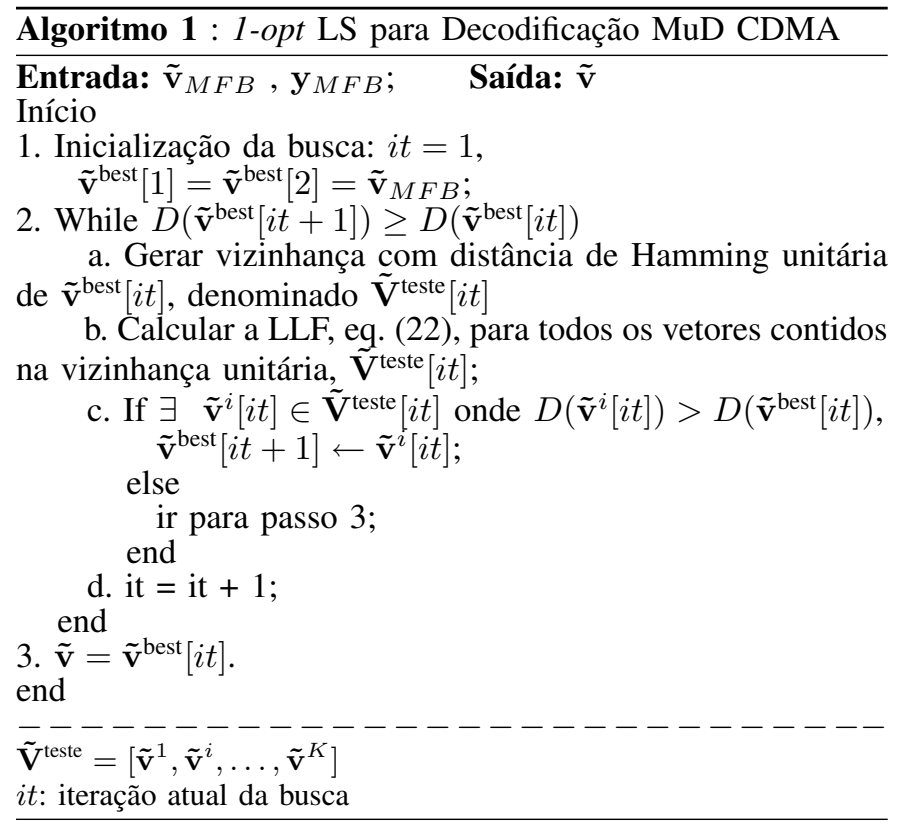

\section{Resultados NuméRicos}

Nesta seção são apresentados os resultados de desempenho para o sistema DS/CDMA codificado proposto, o qual utiliza o algoritmo de busca local no processo de decodificação multiusuário. Os principais valores de parâmetros de sistema, para todas as simulações Monte Carlo, são indicados na tabela I. Além disso, considerou-se um número mínimo de erros/ponto = 15 e controle perfeito de potência entre os usuários.
Para a codificação convolucional, foram utilizados os valores relativos ao padrão CDMA2000 [15] propostos pela IS2000 (IS - Interim Standard), cujo comprimento de restrição possui valor 9, resultando em oito registradores, taxa de codificação $R_{c}=1 / 3$, polinômios geradores definidos por $g_{0}=557, g_{1}=663$ e $g_{2}=711$ em octal, bloco do entrelaçamento (interleaver) dos bits de informação codificados formados por uma matriz de 576 células, sendo 32 linhas e 18 colunas, com entrada por coluna e saída por linha, enquanto o desentrelaçador (deinterleaver) é formado por uma matriz com 18 linhas e 32 colunas de modo a desentrelaçar os bits codificados.

TABELA I

PRINCIPAIS PARÂMETROS DO SISTEMA DS/CDMA COM CODIFICAÇÃO CONVOLUCIONAL E DECODIFICAÇÃo MULTIUSUÁRIO BASEADO EM BUSCA LOCAL 1-opt LS.

\begin{tabular}{|l|c|}
\hline Modulação & BPSK \\
\hline Seqüências de Espalhamento & Aleatória, $N_{s}=31$ \\
\hline Tipo de Codificação & Convolucional \\
\hline Taxa de Codificação & $1 / 3$ \\
\hline Comprimento de Restrição & 9 \\
\hline Polinômio Gerador & $(557,663,711)_{8}$ \\
\hline Comprimento do Traceback & 27 \\
\hline Número de Bits de Quantização & 6 \\
\hline Tamanho do Frame & 192 \\
\hline Duração do Frame & $20 \mathrm{~ms}$ \\
\hline Freqüência da Portadora & $2 \mathrm{GHz}$ \\
\hline Tamanho do Entrelaçador & 576 \\
\hline Algoritmo Heurístico & 1 -opt $\mathrm{LS}$ \\
\hline
\end{tabular}

Para os parâmetros do canal, adotou-se o modelo de Jakes modificado [16], com freqüência da portadora $f_{c}=2 \mathrm{GHz}$, número de osciladores $N_{d}=36$, velocidades de deslocamento dos móveis uniformemente distribuídas entre 0 e $120 \mathrm{~km} / \mathrm{h}$ e número de usuários ativos $K=[1,10,15,20,25,31]$, i.e., carregamento do sistema na faixa de $0,0323 \leq K / N_{s} \leq 1$.

Utilizou-se a relação sinal-ruído média no receptor no intervalo $\bar{\gamma}=[0 ; 24] \mathrm{dB}$, tanto para decodificação de Viterbi utilizando algoritmo de busca local 1-LS com saída suave e abrupta, como para o decodificador de Viterbi na ausência do algoritmo heurístico.

No cômputo da decodificação de Viterbi procurou-se ajustar o valor do parâmetro de entrada denominado de comprimento do traceback (tblen - traceback length), o qual está associado ao número de percursos sobreviventes dos estados correntes da treliça, igual a no máximo 5 vezes o comprimento de restrição (também denominado comprimento de constrição) do polinômio gerador do código convolucional utilizado [17]. Tal parâmetro está associado às transições armazenadas em memória. Visando otimizar este parâmetro, cujo crescimento acarreta diretamente no incremento da capacidade de armazenamento em memória, foram realizadas simulações para a determinação de um valor de melhor compromisso. A Fig. 2 apresenta a melhoria de desempenho à medida que o comprimento do traceback aumenta (aumento do requisito de memória). A melhor escolha em termos do compromisso desempenho $\times$ requisito de memória, consiste em adotar tblen $=27$, i.e., um valor 3 vezes o comprimento de restrição: tblen $=3 \times 9=27$. Note-se que tal escolha representa uma econômia de processamento em relação à topologia proposta 
em [17], uma vez que o total de percursos sobreviventes foi reduzido de 5 vezes [17] para 3 vezes o valor do comprimento de restrição com o uso da estratégia 1-opt LS.

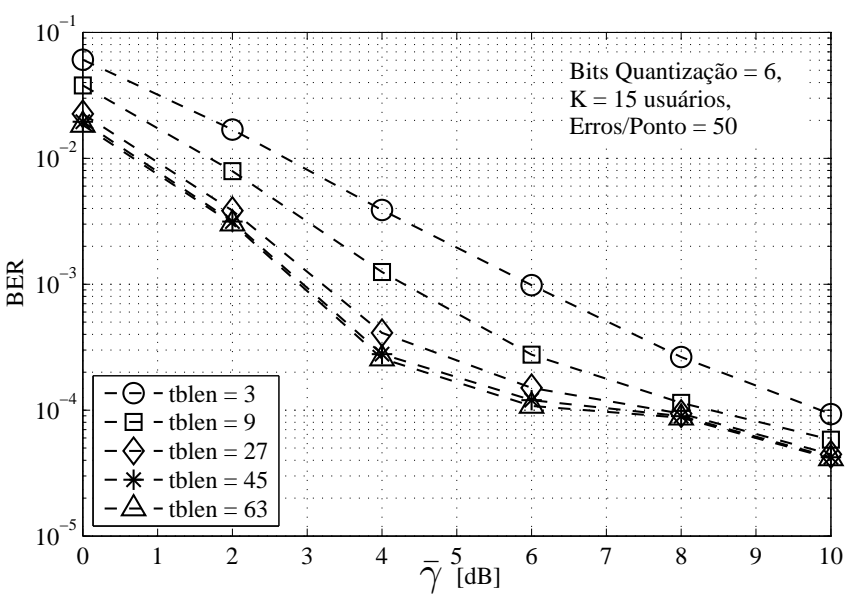

Fig. 2. Número de percursos sobreviventes para a decodificação suave (soft) e abrupta (hard) com 1-opt LS.

O número de bits de quantização $(B Q)$ é outro parâmetro que afeta diretamente a qualidade da decodificação suave de Viterbi, já que ao contrário da decodificação com decisão abrupta, na qual os sinais são quantizados em apenas dois níveis (zeros e uns), a adoção da quantização do sinal em múltiplos níveis implica em um ganho no processo de decodificação. Novamente, buscou-se um valor de compromisso (incremento na complexidade $\times$ melhoria de desempenho) para o parâmetro $B Q$. Observando-se os desempenhos decorrentes da utilização dos valores $B Q=3,5,6,8,10$, concluiu-se que não houve ganho de desempenho significativo para $B Q>6$. Portanto, adotou-se $B Q=6$ para as demais figuras de desempenho desta seção.

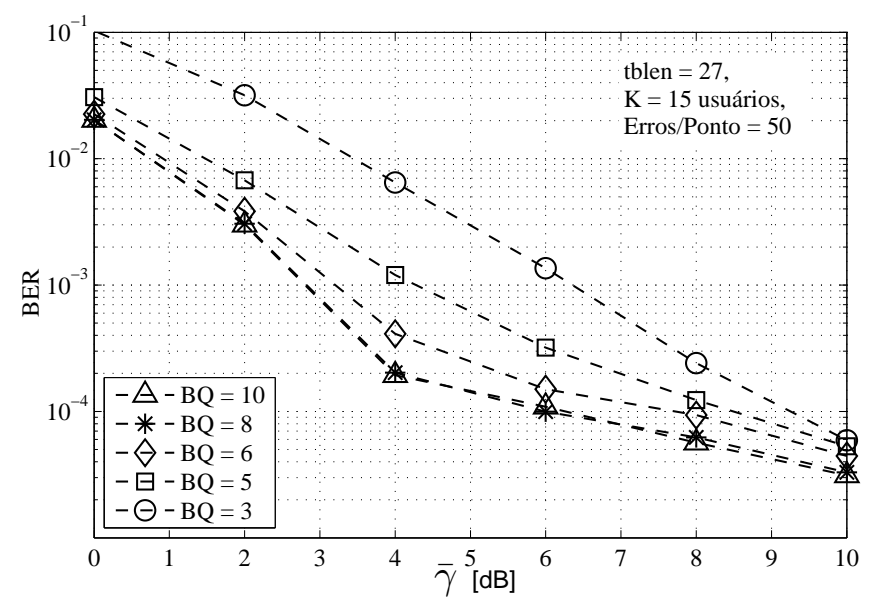

Fig. 3. Número de bits de quantização para a decodificação suave de Viterbi.

A Fig. 4 apresenta o desempenho em termos da taxa de erro de bit média (BER) do decodificador soft na presença e ausência do algoritmo de busca local 1-opt LS, em função da relação sinal-ruído média, $\bar{\gamma}$, sendo parametrizado pelo carregamento do sistema $\left(K / N_{s}\right)$. Note-se que à medida que o número de usuários é incrementado, o desempenho do sistema sofre apreciável degradação quando na ausência do algoritmo 1-opt LS (Fig. 4.a). No entanto, com a utilização do algoritmo de busca local 1-opt, Fig. 4.b, nota-se uma melhoria significativa no desempenho em relação ao método convencional de decodificação soft, quando o carregamento do sistema aumenta. Note-se que para a condição $\bar{\gamma}=12$ dB e carregamento igual a $100 \%$ ( $K=31$ usuários) da Fig. 4.a, o sistema atinge $B E R=1,3 \times 10^{-3}$ com o uso da decodificação convencional. Porém, empregando-se a técnica 1-opt LS, Fig. 4.b, nas mesmas condições, o sistema atinge $B E R=5,6 \times 10^{-5}$, um incremento de desempenho em termos de BER da ordem de $\approx 1,6$ décadas, indicando que o desempenho do decodificador suave de Viterbi sem a técnica 1-opt LS é extremamente sensível ao aumento da MAI.

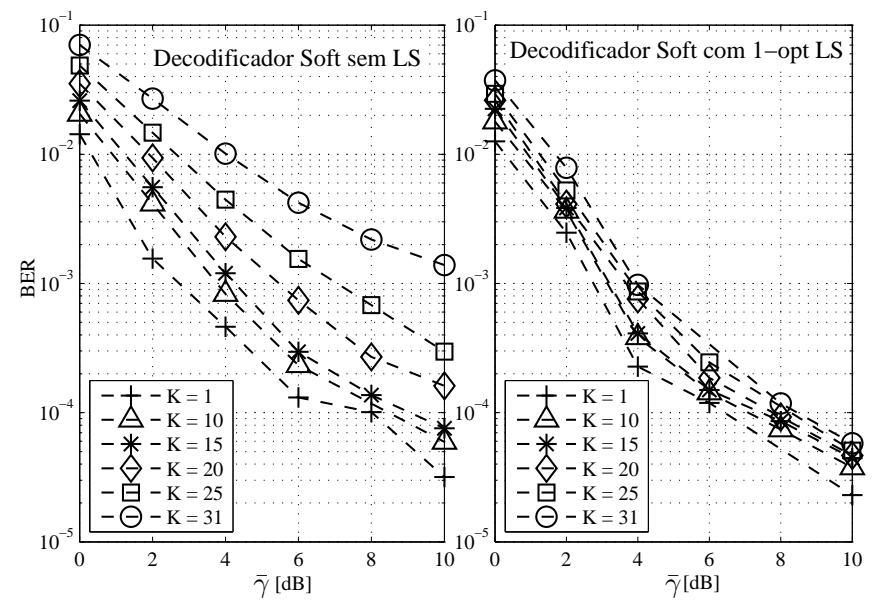

Fig. 4. Desempenho para a decodificação suave de Viterbi em canais Rayleigh plano sem a utilização e com a utilização do 1-opt LS.

A Fig. 5 compara a robustez da estratégia proposta em relação ao aumento do carregamento, com e sem 1-opt LS, considerando decisões soft ou hard e $\bar{\gamma}=8 \mathrm{~dB}$. Observe-se que na presença da estratégia 1-opt LS, a inclinação das duas curvas (decisões soft e hard) é praticamente zero, indicando que o desempenho do sistema é pouco afetado pelo aumento da interferência de múltiplo acesso. Portanto, o emprego de técnicas heurísticas na decodificação convolucional de Viterbi mostra-se efetiva e robusta à interferência de múltiplo acesso, ao passo que na ausência da etapa 1-opt LS de decodificação, o desempenho do sistema é sensivelmente degradado para a decisão hard em toda a faixa de carregamento e para a decisão soft a partir de $50 \%$ de carregamento.

A Fig. 6 apresenta a melhoria de desempenho da estratégia proposta em relação à estratégia convencional de decodificação convolucional em função do aumento da relação sinal-ruído. Considerou-se a situação de pior caso para a MAI, ou seja, carregamento de $100 \%(K=31)$. Nesta condição, nota-se que o desempenho da estratégia proposta é próximo da situação de melhor caso, ou seja, ausência de MAI $(K=1)$ para ambos os tipos de decodificação (soft e hard). No entanto, observase que na ausência do algoritmo 1-opt LS, o desempenho obtido pelo decodificador é extremanente degradado quando o número de usuários aumenta de $K=1$ para $K=31$, evidenciando a aplicabilidade da estratégia proposta. 


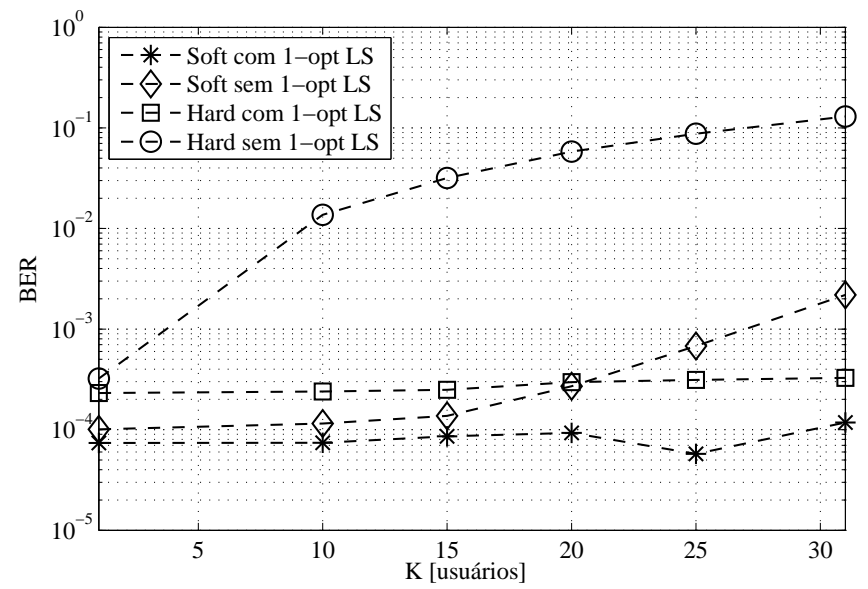

Fig. 5. Desempenho em função do carregamento para $\bar{\gamma}=8 \mathrm{~dB}$ em canais Rayleigh plano.

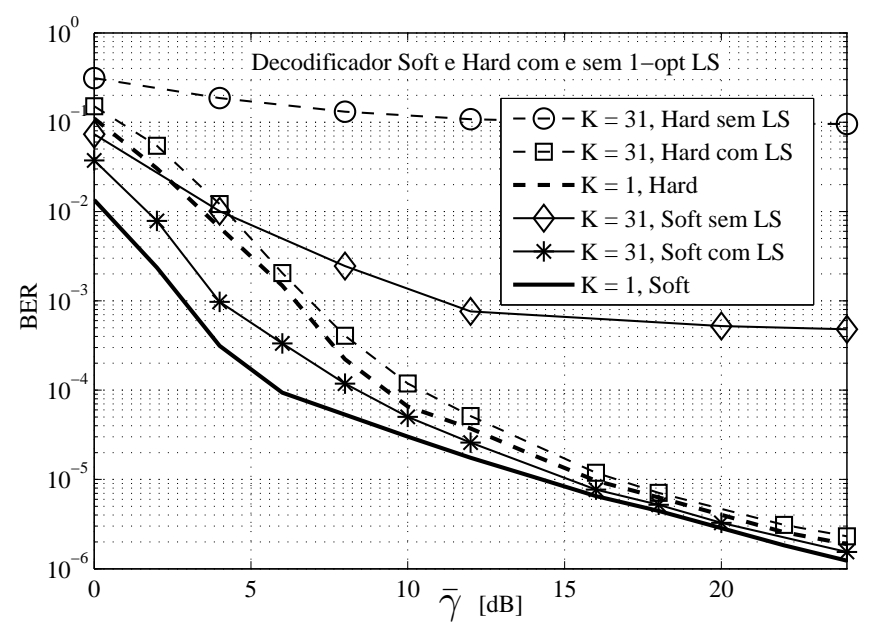

Fig. 6. Comparação dos desempenho para a decodificação soft e hard de Viterbi para canais Rayleigh plano com e sem a utilização do 1-opt LS.

\section{Complexidade Computacional}

Visando demonstrar a viabilidade de implementação da técnica proposta, a Fig. 7 apresenta o acréscimo no número de operações e no requisito de memória quando utiliza-se a técnica 1-opt LS no processo de decodificação comparadoa com a complexidade e o requisito de memória necessários à decodificação convencional, ou seja, utilizando somente o decodificador de Viterbi. Note-se que o acréscimo máximo no número de operações é de $\approx 3 \%$ para $K=1$ e de $\approx 0,06 \%$ para $K=31$, evidenciando um acréscimo marginal de complexidade no processo de decodificação. Além disso, através da Fig. 7 observa-se que o acréscimo percentual no número de operações diminui com o aumento do número de usuários, garantindo a utilização da técnica proposta em sistemas atuais. Por fim, note-se que o acréscimo no requisito de memória é inferior a $0,03 \%$ para $K=1$ e inferior a $0,001 \%$ para $K=31$, evidenciando, novamente, um aumento marginal nos requistos do sistema (processamento e memória).

\section{CONCLUSÕES}

Resultados de desempenho via simulação Monte-Carlo da seção $\mathrm{V}$, referentes à utilização da técnica de busca local 1-opt, tanto para a decodificação suave como para a decodificação abrupta, indicaram valores para a taxa de erro de bit que se

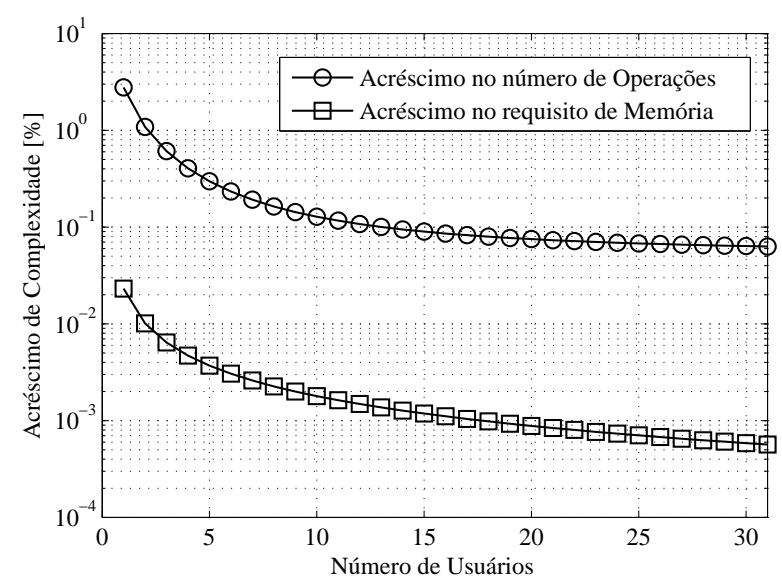

Fig. 7. Acréscimo na complexidade computacional e no requisito de memória, em [\%], quando utiliza-se a técinca 1-opt LS na decodificação.

aproximam do limite uniusuário $(K=1)$, principalmente para as regiões de médio e alto carregamento.

Adicionalmente, considerando ainda a baixíssima complexidade computacional e requisito de memória adicional na utilização do algoritmo 1 -opt LS, comprovou-se, neste trabalho, a aplicabilidade e eficiência desta técnica no processo de decodificação em sistemas DS/CDMA com codificação convolucional.

\section{REFERÊNCIAS}

[1] S. Verdú, Multiuser Detection, New York: Cambridge University Press, 1998.

[2] A. Duel-Hallen, J. Holtzman e Z. Zvonar, "Multiuser Detection for CDMA Systems", IEEE Personal Communications, pp. 46-58, 1995.

[3] H. V. Poor e S. Verdu, "Probability of error in mmse multi-user detection", IEEE Transactions on Information Theory, vol.43, no.3, pp. 858-881, 1997.

[4] P. H. Tan, "Multiuser detection in CDMA-Combinatorial optimization Methods", Chalmers University of Technology, Göteborg, Monografia, 93 f., 2001.

[5] T. Abrão, "Canceladores de Interferência Multiusuário Aplicados a Sistemas DS/CDMA de Múltipla Taxa”. Escola Politécnica da Universidade de São Paulo - EPUSP, Tese Doutorado, 364 f., 2001.

[6] F. Ciriaco, T. Abrão e P. J. E. Jeszensky, "DS/CDMA Multiuser Detection with Evolutionary Algorithms", Journal of Universal Computer Science - J.UCS, vol.12, no.4, pp. 450-480, 2006.

[7] C. Xu, R. Maunder, L. Yang e L. Hanzo, "Near-optimum soft-output antcolony-optimization based multiuser detection for the ds-cdma uplink", IEEE International Conference on Communications (ICC 2008). Beijing, China, pp.795-799, 2008.

[8] C. Xu, R. Maunder, L. Yang e L. Hanzo, "Near-Optimum Multiuser Detectors using Soft-Output Ant-Colony-Optimization for the DS-CDMA Uplink", IEEE Signal Processing Letters, v. 16, n. 2, pp.137-140, 2009.

[9] J.G. Proakis, Digital Communications, Fourth Edition. McGraw-Hill, 2000.

[10] T. K. Moon e W. C. Stirling, Mathematical Methods and Algorithms for Signal Processing, Englewood Cliffs, NJ: Prentice-Hall, 2002.

[11] S. Kay, Fundamentals of Statistical Signal Processing, Estimation Theory, New Jersey, U.S.A.: Prentice Hall, 1993.

[12] S. Verdú, "Computational Complexity of Optimum Multiuser Detection", Algorithmica, no. 4, pp.303-312, 1989.

[13] M. C. Goldbarg e H. P. L. Luna, Otimização Combinatória $e$ Programação Linear. Rio de Janeiro: Campus. 2000. 649 p.

[14] C. Reeves, Modern Heuristic techniques for combinatorial problems. Oxford: Blackwell Scientific, 1993.

[15] TIA/EIA/IS-2000-2, "Physical Layer Standard for cdma2000 Spread Spectrum Systems", Telecomunications Industry Association, 1999.

[16] P. Dent, G E. Bottomley e T. Croft, "Jakes fading model revisited", Electron. Lett., vol.29, no.3, pp. 1162-1163, June 1993.

[17] T. H. Liew e L. Hanzo, "Space-Time Block Codes and Concatenated Channel Codes: A Historical Perspective and Comparative Study", Proc. of the IEEE, Febr. 2001. 